\title{
Regulation of fatty acid composition and lipid storage by thyroid hormone in mouse liver
}

\author{
Xuan Yao ${ }^{1,2 \dagger}$, Sarina Hou ${ }^{1 \dagger}$, Duo Zhang ${ }^{1,2}$, Hongfeng Xia ${ }^{1,2}$, Yu-Cheng Wang ${ }^{2,3}$, Jingjing Jiang ${ }^{4}$, Huiyong Yin ${ }^{1,5}$ \\ and Hao Ying ${ }^{1,2,5^{*}}$
}

\begin{abstract}
Background: Thyroid hormones (THs) are potent hormones modulating liver lipid homeostasis. The perturbation of lipid homeostasis is a hallmark of non-alcoholic fatty liver disease (NAFLD), a very common liver disorder. It was reported that NAFLD patients were associated with higher incidence of hypothyroidism. However, whether abnormal thyroid function contributes to the pathogenesis of NAFLD remains unclear.

Results: We used in vivo models to investigate the influence of hypothyroidism and TH on hepatic lipid homeostasis. We did not observe hepatic triglyceride accumulation in the liver of hypothyroid mice, although the liver was enlarged. We then characterized the hepatic fatty acid composition with gas chromatography-mass spectrometry in mice under different thyroid states. We found that hypothyroidism decreased saturated fatty acid (SFA) content while TH treatment restored the level of SFA. In agreement with this finding, we observed that the expression of acetyl-CoA carboxylase 1 and fatty acid synthase, the rate-limit enzymes for de novo lipogenesis (DNL), decreased in hypothyroid mice while increased after TH treatment. We also found that the ratio of C18:1n-9/C18:0 and C16:1n-7/C16:0 was decreased by TH treatment, suggesting the activity of stearoyl-CoA desaturase- 1 was suppressed. This finding indicated that TH is able to suppress triglyceride accumulation by reducing fatty acid desaturation. Additionally, we found that hepatic glycogen content was substantially influenced by TH status, which was associated with glycogen synthase expression. The increased glycogen storage might explain the enlarged liver we observed in hypothyroid mice.
\end{abstract}

Conclusions: Taken together, our study here suggested that hypothyroidism in mice might not lead to the development of NAFLD although the liver became enlarged. However, disturbed TH levels led to altered hepatic fatty acid composition and glycogen accumulation.

Keywords: Thyroid hormone, Liver, Fatty acid, Glycogen, NAFLD

\section{Background}

Thyroid hormone (TH) is potent to influence multiple aspects of lipid, carbohydrate, protein and mineral metabolism [1]. Through binding to nuclear TH receptors (TR), TH can modulate the expression of target genes [2]. Physiological inverse relationship between $\mathrm{TH}$, such as thyroxine (T4) and triiodothyronine (T3), and thyroid-

\footnotetext{
* Correspondence: yinghao@sibs.ac.cn

${ }^{\dagger}$ Equal contributors

${ }^{1}$ Key Laboratory of Food Safety Research, Institute for Nutritional Sciences, Shanghai Institutes for Biological Sciences, Graduate School of the Chinese Academy of Sciences, Chinese Academy of Sciences, Shanghai 200031, China ${ }^{2}$ Clinical Research Center of Institute for Nutritional Sciences, Shanghai Institutes for Biological Sciences, Chinese Academy of Sciences, Shanghai 200031, China

Full list of author information is available at the end of the article
}

stimulating hormone (TSH) are maintained through a classic negative feedback loop mediated by the hypothalamicpituitary-thyroid axis. When the components of the feedback loop cannot act co-ordinately, it usually indicates incident of diseases. Overt hyperthyroidism is used to describe the situation when patients are found to have an undetectable TSH level and a high T4 or T3 level. In contrast, those who are diagnosed overt hypothyroidism show an elevated TSH level accompanied by a low free T4 level [3]. Subclinical thyroid disease refers to the situation when increased (hypothyroidism) or decreased (hyperthyroidism) plasma TSH concentration is observed while free $\mathrm{T} 4$ and total T3 concentrations are in normal range [4]. Resistance to thyroid hormone (RTH) defines a syndrome 
in which tissues present reduced sensitivity to the action of $\mathrm{TH}[5]$.

The liver plays pleiotropic roles in modulating fatty acid and carbohydrate homeostasis. With abundant energy intake, ATP is preferentially generated through carbohydrate oxidation and glycogen stores are replenished in liver and skeletal muscle. When there is further surplus, the carbohydrate is converted to fatty acids [6], which is referred as de novo lipogenesis (DNL) [7] that gives rise to saturated fatty acids (SFA). Fatty acids also undergo modifications such as elongation and desaturation in liver before they are esterified for storage and secretion. Non-alcoholic fatty liver diseases (NFALD), histologically categorized into non-alcoholic fatty liver (NAFL) and non-alcoholic steatohepatitis (NASH), is a group of diseases characterized by hepatic steatosis without significant alcohol consumption or steatogenic medication [8]. It was reported that there was an enhanced lipogenesis in NAFLD patients [9].

The abnormal TH status is frequently linked to hepatic lipid homeostasis alterations [10]. It was reported that NASH patients had a higher prevalence of hypothyroidism than the control group [11]. Also, the severity of NAFLD is associated with the grade of hypothyroidism in a dosedependent manner [12]. Moreover, TR $\beta$ PV/PV mice, a mouse model of RTH due to its impaired TR $\beta$ function, developed fatty liver [13]. Hepatic steatosis was observed in a TR $\alpha$ dominant-negative $\mathrm{P} 398 \mathrm{H}$ mutation [14]. However, these studies cannot answer the question that whether abnormal TH level directly contributes to the pathogenesis of NAFLD.

Although much has been learnt about the molecular mechanisms of THs regulating gene expression involved in lipogenesis [15], little is known about the effect of disturbed THs on hepatic lipid storage and fatty acid composition. Deranged hepatic fatty acid compositions may reflect disturbed lipogenesis, elongation and desaturation. How $\mathrm{TH}$ effects on lipogenesis impact the overall picture of hepatic fatty acid remains to be elucidated in detail.

Since it has been reckoned that $\mathrm{TH}$ positively regulates genes involved in hepatic lipogenesis, the use of TR agonists for the treatment of NAFLD has been theoretically considered unviable. However, a bunch of TR agonists have been implicated to potently treat hepatic steatosis [16] or reduce hepatic triglyceride [17]. In addition, the proposed positive regulation of hepatic lipogenesis could not be used to explain the fatty liver developed in TR $\beta P V / P V$ mice. These suggested that the complex mechanism involved in $\mathrm{TH}$ regulating liver lipid homeostasis is still poorly elucidated.

In this study, we aimed to figure out the roles of $\mathrm{TH}$ in hepatic lipid homeostasis. To see if the mice with manipulated $\mathrm{TH}$ status recapitulated the features observed in NAFLD patient and animal models, we characterized the liver of our mouse model mainly in terms of lipid accumulation. Taking advantage of the gas chromatographymass spectrometry (GC-MS) methods, we also quantified the hepatic fatty acid composition. At the same time, we determined the expression levels of genes that control the key steps of DNL and fatty acid modification. Collectively, these findings may help to uncover the relationship between NAFLD and disturbed TH level.

\section{Results}

\section{Liver weight and hepatic lipid content}

To investigate whether abnormal TH levels would lead to hepatic features similar to NAFLD, we treated mice with methimazole (MMI) so as to render them hypothyroidism (hypothyroid mice) (Additional file 1: Figure S1) [18]. We found that the MMI treatment led to $27.41 \%$ increase in liver-to-body weight ratio in hypothyroid mice compared with control mice $(6.88 \pm 0.06 \%$ v.s. $5.40 \pm 0.02 \%, \mathrm{p}<0.05$, $\mathrm{n}=5$ in each group) (Figure 1A). To find out the cause for the liver weight gain, we first measured the hepatic triglyceride contents to see if steatosis, a hallmark of NAFLD [19], led to liver weight alteration. However, we found that the hepatic triglyceride content was significantly reduced in hypothyroid mice compared with control mice (Figure 1B). Neither did oil-red $\mathrm{O}$ staining show hepatic lipid accumulation in hypothyroid mice (Figure 1C). Moreover, H\&E staining showed no sign of ballooning

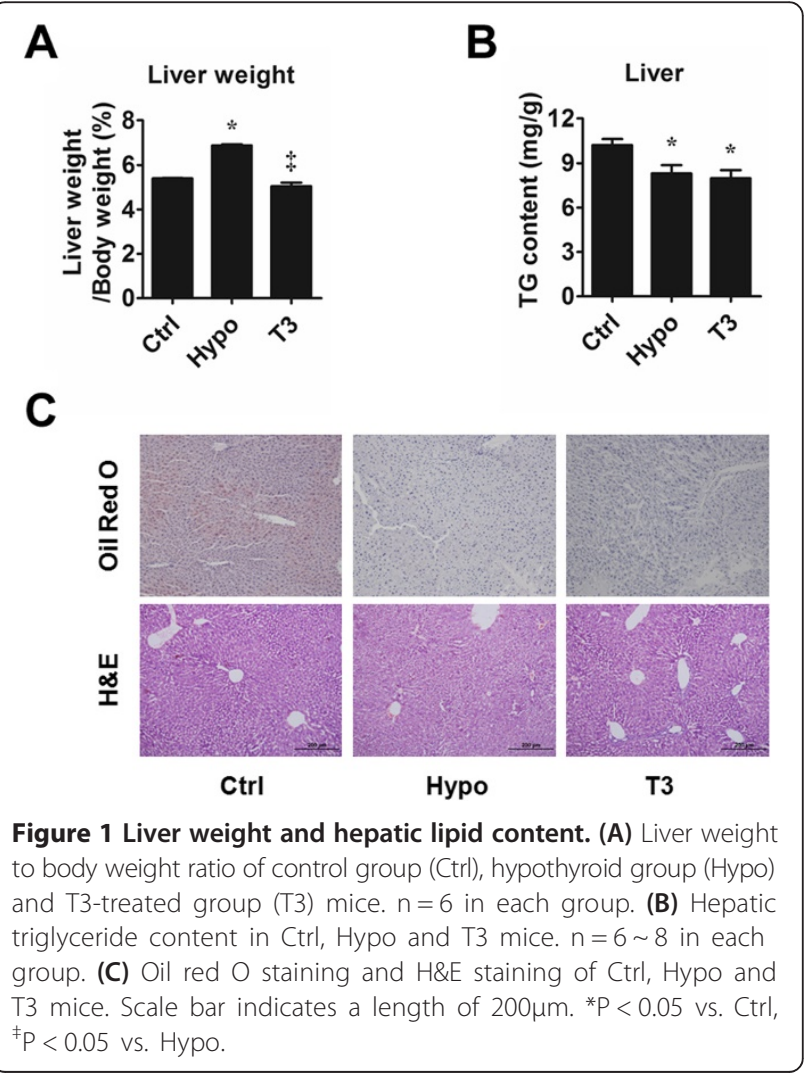


in the liver of hypothyroid mice, one of the histological features of NASH (Figure 1C). Collectively, the decreased hepatic lipid accumulation and histological features found in our hypothyroid mice did not recapitulate that of NAFLD.

To find out how TH affects hepatic lipid homeostasis, we treated some mice in hypothyroid group with T3 for 5 days (Additional file 2: Figure S2). We confirmed the effect of T3 treatment by the elevated hepatic type 1 iodothyronine deiodinase (Dio1) level (Additional file 1: Figure S1), a known sensitive marker of peripheral thyroid status [20]. It was observed that T3 treatment resulted in $26.74 \%$ decrease in liver-to-body weight ratio compared with that of hypothyroid mice (Figure 1A) $(5.04 \pm 0.18 \%$ v.s. $6.88 \pm 0.06 \%, \mathrm{p}<0.05, \mathrm{n}=5$ in each group). In T3treated mice, the hepatic triglyceride and lipid accumulation were also lower than that of control mice (Figure 1B \& C). Besides, no ballooning was observed in T3-treated mice as manifested by H\&E staining (Figure 1C). In sum, in our mouse model, the disturbed TH level led to liver weight alteration. Also, hepatic lipid accumulation decreased in hypothyroid and T3-treated mice compared with that of the control.

Hepatic lipogenesis and saturated fatty acid compositions Hepatic de novo lipogenesis (DNL) contribute to the lipid content alteration. To investigate if the hepatic DNL was altered under different thyroid hormone status, we compared the expression of two key enzymes involved in DNL, Acetyl-CoA carboxylase 1 (ACC1) and Fatty acid synthase (FASN), between the three groups. It was observed that compared with control mice, ACC1 and FASN decreased on the protein levels in hypothyroid mice; when treated with T3, the protein levels of ACC1 and FASN was restored to a level comparable to the control mice (Figure 2). Compared with the control mice, the mRNA level of ACC1 was increased in hypothyroid mice while it was not significantly reduced in T3-treated mice. As for FASN mRNA, there was no significant difference between control and hypothyroid mice while it was lower in T3-treated mice (Additional file 3: Figure S3). The altered gene expression prompted us to investigate whether the products of DNL in the liver would also be affected by TH status. Since the DNL products belong to the saturated fatty acid (SFA) family, we measured and compared the SFA amount between the three groups (Table 1). The major products of DNL, C16:0 and C18:0 [21,22], decreased significantly in the liver of hypothyroid mice liver compared with control mice (Table 1). The trend among the rest members of SFA was consistent and qualitatively similar across the family, suggesting that the DNL in hypothyroid mice was suppressed. Thus, we proposed that depressed hepatic DNL partly contributed to the decreased lipid accumulation in the liver of hypothyroid mice.

On the other hand, in T3-treated mice, it was observed that the levels of the SFA concentration were higher than that of hypothyroid mice (Table 1), which indicated an enhanced hepatic DNL in response to T3 treatment. This was also in consistent with the reduced ACC1 and FASN protein level in hypothyroid mice and increased one in T3-treated group (Figure 2).

\section{Hepatic unsaturated fatty acid (UFA) compositions and Stearoyl-CoA desaturase (SCD-1)}

Fatty acids also undergo modifications such as desaturation before they were esterified into triglyceride. Therefore, we also quantified the individual monounsaturated fatty acid (MUFA), the products of desaturation, in the liver from the three groups. As shown in Table 2, the significantly reduced level of C18:1n-9, a most abundant MUFA in the liver, rendered total MUFA concentration lower in T3-treated mice than the control mice. It is known that in the liver, Stearoyl-CoA desaturase (SCD-1) is responsible for the conversion of $\mathrm{C} 16: 0$ and $\mathrm{C} 18: 0$ into C16:1n-7 and C18:1n-9, respectively. Thus, we compared the ratios between the products and the precursors and use them as surrogate measures to determine the SCD-1 activity. As shown in Figure 3A, the ratio of C18:1n-9/ C18:0 indicated a significantly depressed SCD-1 activity in T3-treated mice compared with the control mice. Although not reaching a statistical significance, the alteration of C16:1n-7/C16:0 showed a similar trend with C18:1n-9/C18:0 (Figure 3A). In agreement with this, we found that the SCD-1 in T3-treated mice was significantly

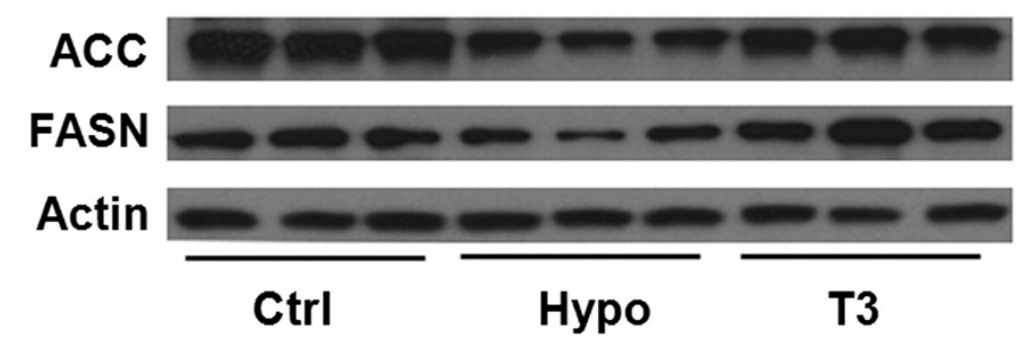

Figure 2 Hepatic ACC1 and FASN expression levels. Western blotting analysis of ACC1 and FASN in liver from control group (Ctrl), hypothyroid group (Hypo) and T3-treated group (T3). 
Table 1 Liver saturated fatty acid (SFA) quantification from different groups

\begin{tabular}{llll}
\hline Fatty acid & Control & Hypothyroid & T3-treated \\
\hline C14:0 & $57.9 \pm 18.4$ & $45.1 \pm 11.3$ & $25.5 \pm 1.5$ \\
C16:0 & $17093 \pm 1256$ & $13271 \pm 658^{*}$ & $15322 \pm 720^{\ddagger}$ \\
C18:0 & $9769 \pm 861$ & $7612 \pm 424^{*}$ & $12566 \pm 1013^{* \neq}$ \\
C20:0 & $284 \pm 27$ & $132 \pm 12^{*}$ & $214 \pm 18^{* \neq}$ \\
C22:0 & $254 \pm 22$ & $126 \pm 10^{*}$ & $374 \pm 19^{* \neq}$ \\
C24:0 & $164 \pm 18$ & $139 \pm 5$ & $191 \pm 17^{\ddagger}$ \\
SFA & $27623 \pm 1631$ & $21762 \pm 937^{*}$ & $28687 \pm 1700^{\ddagger}$
\end{tabular}

Individual fatty acid amount was measured by GC-MS and normalized to liver weight $(\mathrm{nmol} / \mathrm{g})$. Values represent the mean \pm S.E. from five mice in the same group.

${ }^{*} \mathrm{P}<0.05$ vs. Control group

${ }^{\ddagger} \mathrm{P}<0.05$ vs. Hypothyroid group.

Table 2 Liver unsaturated fatty acid (UFA) quantification from different groups

\begin{tabular}{|c|c|c|c|}
\hline Fatty acid & Control & Hypothyroid & T3-treated \\
\hline C16:1n-7 & $862 \pm 135$ & $660 \pm 84$ & $647 \pm 46$ \\
\hline C16:1n-9 & $191 \pm 22$ & $164 \pm 26$ & $95 \pm 16^{*}$ \\
\hline C18:1n-7 & $1453 \pm 156$ & $1647 \pm 228$ & $1209 \pm 117$ \\
\hline C18:1n-9 & $7754 \pm 547$ & $6789 \pm 742$ & $5687 \pm 421^{*}$ \\
\hline C20:1n-9 & $282 \pm 223$ & $222 \pm 26$ & $151 \pm 9^{*}$ \\
\hline$C 22: 1 n-9$ & $88.1 \pm 20.6$ & $50.45 \pm 13.1$ & $34.0 \pm 2.6^{*}$ \\
\hline$C 24: 1 n-9$ & $144 \pm 15$ & $169 \pm 10$ & $111 \pm 10^{\ddagger}$ \\
\hline MUFA & $10774 \pm 825$ & $9702 \pm 1076$ & $7933 \pm 605^{*}$ \\
\hline C18:3n-3 & $231 \pm 23$ & $186 \pm 21$ & $207 \pm 19$ \\
\hline$C 20: 5 n-3$ & $1174 \pm 111$ & $1241 \pm 79$ & $2137 \pm 187^{* \neq}$ \\
\hline$C 22: 5 n-3$ & $653 \pm 51$ & $707 \pm 56$ & $898 \pm 53^{*^{\ddagger}}$ \\
\hline$C 22: 6 n-3$ & $10200 \pm 972$ & $9479 \pm 421$ & $10362 \pm 649$ \\
\hline n-3 PUFA & $12366 \pm 1105$ & $11857 \pm 571$ & $13722 \pm 887$ \\
\hline$C 18: 2 n-6$ & $13664 \pm 970$ & $14302 \pm 853$ & $13829 \pm 820$ \\
\hline C18:3n-6 & $231 \pm 23$ & $186 \pm 21$ & $207 \pm 19$ \\
\hline$C 20: 2 n-6$ & $249 \pm 23$ & $268 \pm 28$ & $210 \pm 13$ \\
\hline C20:3n-6 & $1355 \pm 125$ & $1807 \pm 181$ & $1201 \pm 121^{\ddagger}$ \\
\hline$C 20: 4 n-6$ & $5795 \pm 646$ & $5630 \pm 396$ & $6996 \pm 733$ \\
\hline$C 22: 4 n-6$ & $105 \pm 14$ & $93 \pm 12$ & $94 \pm 11$ \\
\hline n-6 PUFA & $21398 \pm 1728$ & $22286 \pm 1385$ & $22536 \pm 1613$ \\
\hline PUFA & $33764 \pm 2822$ & $34144 \pm 1952$ & $36258 \pm 2485$ \\
\hline C18:1t & $29.2 \pm 6.3$ & $72.9 \pm 17.8$ & $25.8 \pm 6.1$ \\
\hline $18: 2 n-6.9 c 12 t$ & $27.7 \pm 1.4$ & $39.0 \pm 4.8$ & $34.0 \pm 4.5$ \\
\hline $18: 2 n-6.9 t 12 c$ & $27.2 \pm 6.6$ & $68.6 \pm 16.5^{*}$ & $13.2 \pm 1.31^{* \neq}$ \\
\hline TFA & $84.2 \pm 9.5$ & $180.5 \pm 30.1^{*}$ & $73.0 \pm 11.0^{\ddagger}$ \\
\hline
\end{tabular}

Individual fatty acid amount was measured by GC-MS and normalized to liver weight $(\mathrm{nmol} / \mathrm{g})$. Values represent the mean \pm S.E. from five mice in the same group.

*P $<0.05$ vs. Control group.

${ }^{\ddagger} \mathrm{P}<0.05$ vs. Hypothyroid group. suppressed on the transcriptional and translational level (Figure 3B and C). In hypothyroid mice, we did not find significant difference in SCD-1 activity compared with control mice in regard of the surrogate measures and mRNA levels (Figure 3A \& B).

As for $\mathrm{n}-3$ and $\mathrm{n}-6$ polyunsaturated fatty acids (PUFAs), we did not observe significant differences between control and hypothyroid mice (Table 2). In the T3-treated mice, hepatic C20:5-3 and C22:5n-3 concentration significantly increased compared with control and hypothyroid mice. C20:3n-6 concentration reduced in T3-treated mice compared with hypothyroid mice (Table 2). Despite the statistically insignificant alteration of C18:1t and C18:2n6.9c12t, the trans fatty acid (TFA) we detected showed similar patterns of change where the concentration rose in hypothyroid mice and decreased in T3-treated mice (Table 2).

When the amount of fatty acids were added together according to the degree of saturation, it was discovered that SFA level trended downward in hypothyroid mice while in T3-treated mice it was indistinguishable from that of the control mice (Figure 3D). The MUFA content was significantly lower in T3-treated mice than the other two groups (Figure 3D). As is known, both SFA and MUFA are able to be incorporated into triglyceride. However, MUFAs are the more favored the substrates [23,24]. The reduced MUFA level in T3-treated mice might have offset the effect of elevated SFA level in enhancing triglyceride synthesis. Consequently, the triglyceride content was still lower in T3-treated mice compared with the control mice. $\mathrm{n}-3$ PUFA did not show significant alteration among different groups (Figure 3D). On the other hand, n-6 PUFA increased in hypothyroid mice and decreased in T3-treated mice compared with the control (Figure 3D). We also found that compared with the control mice, the $n-6 / n-3$ PUFA ratio significantly elevated in hypothyroid mice and was the lowest in the T3-treated mice (Figure 3E).

\section{Hepatic glycogen content}

Since the triglyceride level could not well explain the liver weight alteration among the groups, we further determined the content of hepatic glycogen, a frequently variable component of liver. It was observed that in hypothyroid mice the glycogen content was higher than the control, whereas in T3-treated mice the glycogen content was lower than both control and hypothyroid mice (Figure 4A). PAS staining displayed a similar result (Figure 4B). In consistency, the expression of liver glycogen synthase (GYS2), the enzyme catalyzing the rate-limiting step in the synthesis of glycogen, increased in hypothyroid mice and decreased in T3-treated mice compared with the control (Figure 4C \& D). The inverse relationship between GYS2 expression and thyroid hormone level indicated that it was possible GYS2 expression could be regulated 
A

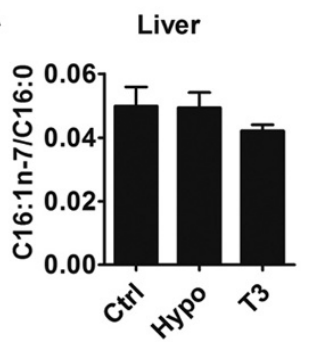

C

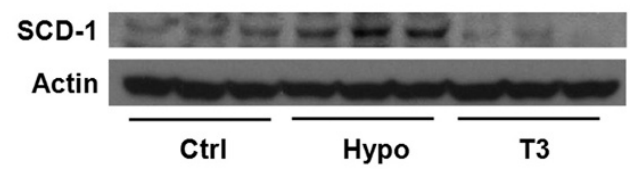

D

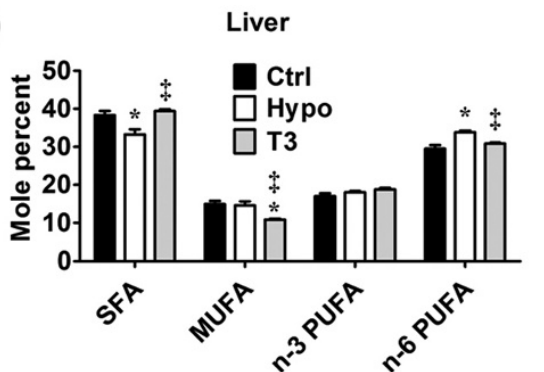

B

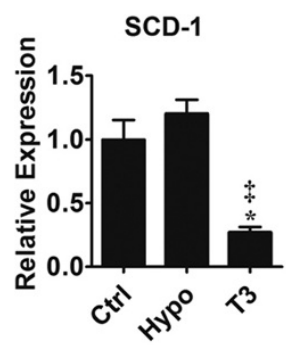

E

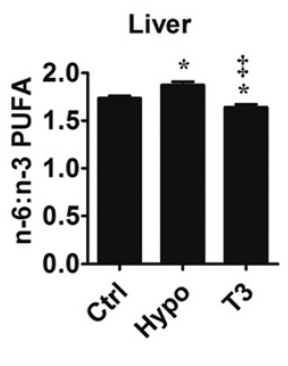

Figure 3 Unsaturated fatty acid (UFA) concentration and SCD-1 expression. (A) C16:1n-7/C16:0 and C18:1n-9/C18:0 ratios were compared and used as surrogate measures of hepatic SCD-1 activity. $n=5$ in each group (B) RT-PCR analysis of SCD-1 gene expression in mouse liver from control group (Ctrl), hypothyroid group (Hypo) and T3-treated group (T3). 18s amplified in parallel served as internal reference. $n=6$ in each group. (C) Western blotting analysis of SCD-1 with $\beta$-Actin as an indication of equal protein loading. (D) Fatty acids were grouped according to the degree of saturation and the amount of fatty acid belonging to the same group was added up. The relative contents of each group were compared across the Ctrl, Hypo and T3. $n=5$ in each group. (E) The ratios between $n-6$ PUFA and $n-3$ PUFA in different groups were compared. $n=5$ in each group. *P $<0.05$ vs. Ctrl, ${ }^{\ddagger} \mathrm{P}<0.05$ vs. Hypo.

by $\mathrm{TH}$ on the transcriptional level. To prove this, luciferase assasy was performed. However, we did not found the fragment we clone from GYS2 promoter responded to T3 (Additional file 4: Figure S4). In sum, we proposed that glycogen content differences might partly account for the liver weight alteration.

\section{Discussion}

T3 is the active form of $\mathrm{TH}$, a well known hormone for its regulation of cellular and tissue metabolism. Through binding to its cognate nuclear receptors with ligandinducible property, T3 controls a broad range of gene expression in target tissues including liver. The presence of T3 enables TRs to bind to TH response elements in the promoters of target genes and form co-activator complexes to activate transcription [15]. Although studies reported that $\mathrm{TH}$ and TRs are potent to control genes involved in lipid and carbohydrate metabolism on transcription level $[25,26]$, they did not necessarily represent the metabolic consequence of the altered TH levels. Our study presents the quantities of major fatty acids within the liver from mice under different $\mathrm{TH}$ status. Along with it we also showed that liver glycogen accumulation was affected by $\mathrm{TH}$ levels. Both of this provides some novel insights into the picture of liver metabolism under disturbed TH levels.

We did not observe increased triglyceride accumulation, the hallmark of NAFLD, in MMI-induced hypothyroid mice, although the hypothyroid mice showed enlarged livers. In contrast, we found that the main fatty acid products of DNL along with the overall SFA concentration fell significantly in hypothyroid mice, which indicated a depressed fatty acid synthesis in liver. The decreased ACC1 and FASN protein level caused the downward regulated DNL to a great extent. One point worth mentioning is that the mRNA level of ACC1 and FASN in T3-treated mice did not increase. The possible reason was that in our research, T3-treated mice were euthanized $24 \mathrm{~h}$ later after the last T3 injection, at which their serum T3 level was not significantly different from that of control mice (Additional file 1: Figure S1A) due to the short half life of serum T3 [20]. As a result, the ACC1 and FASN transcription was not elevated in T3-treated group compared with the control. The lack of triglyceride accumulation in our hypothyroid mouse model suggested that 


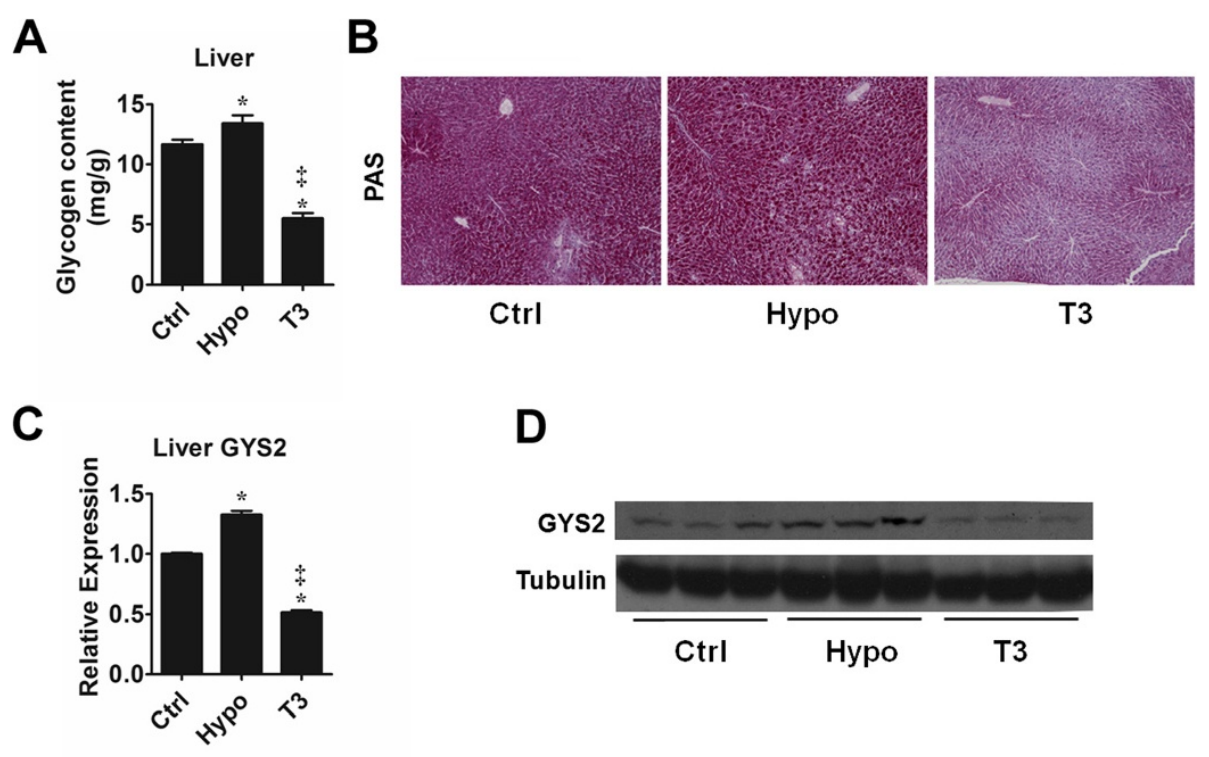

Figure 4 Hepatic glycogen content and the expression of gene related to glycogen synthesis. (A) Hepatic glycogen content was measured from liver samples obtained in group control group (Ctrl), hypothyroid group (Hypo) and T3-treated group (T3). $n=6$ in each group. (B) PAS staining of liver section from Ctrl, Hypo and T3 indicated the presence of glycogen. (C) RT-PCR analysis of GYS2 gene expression in mouse liver from Ctrl, Hypo and T3. 18s amplified in parallel served as internal reference. $n=6 \sim 8$ in each group. (D) Western blotting analysis of GYS2 with a-Tubulin as an indication of equal protein loading. ${ }^{*} \mathrm{P}<0.05$ vs. Ctrl, ${ }^{\ddagger} \mathrm{P}<0.05$ vs. Hypo.

hypothyroidism caused by MMI may not cause the steatosis in NAFLD. We could not rule out the possibility that MMI-induced hypothyroidism model and clinically diagnosed hypothyroidism are different. Thus, population based cohort studies are required to confirm our result.

When comparing the T3-treated mice to the hypothyroid mice, we observed elevated DNL product accumulation as well as gene expression. However, no clue indicated enhanced triglyceride accumulation in the T3-treated mice. The main reason for this was that the depressed SCD-1 function led to decreased MUFA, the other critical component of triglyceride (Figure 5). The lack of triglyceride accumulation in T3-treated mice also indicated the possibility of an enhanced fatty acid oxidation which overriding the effect of DNL. It has been proved in various studies that T3 can positively affect genes involved in fatty acid oxidation $[27,28]$ while in hypothyroidism fatty acid oxidation is greatly decelerated. To be noted, the three TFA we detected mainly came from food ingested [29]. Since the food maintained constant gradient throughout experiment, the altered TFA level could be partly ascribed to its oxidation rate. This enables us to propose that the
A

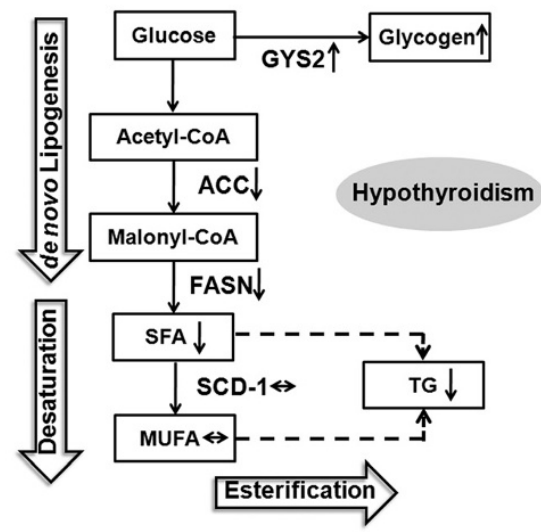

B

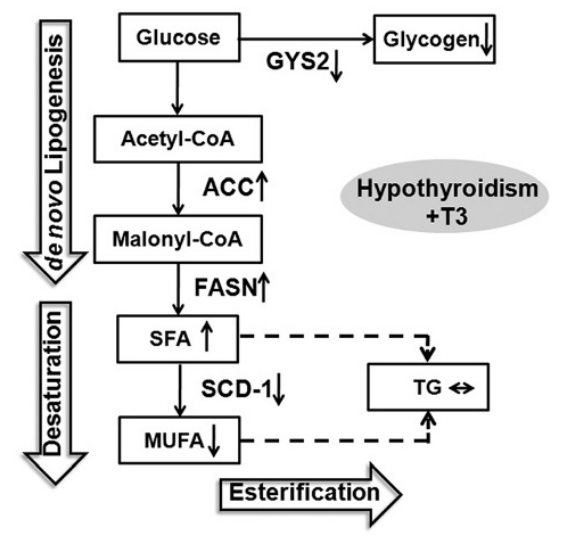

Figure 5 Flow charts illustrating the preferred metabolic pathways under hypothyroid status before and after T3 treatment. The hypothyroidism was compared with Control (A) while hypothyroidism + T3 status was compared with hypothyroidism (B). 
elevated TFA concentration observed in hypothyroid mice could be resulted from decreased rates of fatty acid oxidation (Table 2). Indeed, T3 treatment for 5 days significantly decreased the total TFA concentration compared with hypothyroid mice, indicating enhanced fatty acid oxidation in the T3-treated mice.

Our study further corroborates that SCD-1, the main enzyme responsible of the conversion of SFA to MUFA, was negatively regulated by T3 [30] and [31]. Mice lack SCD-1 exhibited enhanced beta-oxidation [32]. In vivo evidence supported that decreased activity of SCD-1 was linked to favourable outcome of liver in terms of lipid accumulation and secretion. However, there was also study showing that lack of SCD-1 activity would sensitize hepatocyte to SFA-induced apoptosis and render mice lack SCD-1 vulnerable to liver damage other than steatosis [23]. The evidence collectively manifests the importance of relative composition of fatty acids in a defined pool.

The untoward effect like extra hepatic effect greatly impeded T3 application to treat NAFLD. In view of this dilemma, a number of TR agonists with either isoform selectivity or tissue specificity have been developed to lower hepatic triglyceride levels $[16,17,33]$. It is worthwhile to study the effect of TR agonists in modulating DNL and SCD-1 activity so as to set better therapeutic windows.

Similar to the liver in the NAFLD [34], we also observed an increased hepatic $n-6 / n-3$ PUFA ratio in hypothyroid mice, although in a different pool of lipid. In response to $\mathrm{T} 3$ treatment, the ratio decreased to a level even lower than the control mice. Plenty efforts have been spared in addressing what the PUFA ratio indicates. But most of them focused on the ratios in different dietary sources. It still deserves additional research to uncover the indication of increased hepatic $n-6 / n-3$ PUFA ratio.

Another point noteworthy is that hypothyroidism and T3 treatment affect glycogen accumulation, an important aspect of energy metabolism. The clinical relevance of hepatic glycogen content and thyroid disease is not well studied. Previous studies did not uncover the effect of abnormal TH level on the hepatic glycogen accumulation $[35,36]$. Our data showed that glycogen accumulation increased in hypothyroid mice whereas with T3 treatment it decreased significantly. Glycogen is the most instant energy source stored in liver. As noted, DNL was suppressed in hypothyroid mice. This suppression could direct the ingested surplus carbohydrate to store in the form of glycogen (Figure 5). The overall alterations led to an elevated level of hepatic glycogen and declining triglyceride and lipid accumulation. After all, the weight gain and loss in the liver might be partly ascribed to glycogen amount.

\section{Conclusions}

In conclusion, our study provides several novel observations about the alterations in the hepatic fatty acid composition and glycogen storage in mice under disturbed TH status (Figure 5). These observations may provide novel insight into the relationship between $\mathrm{TH}$ and liver metabolism. The results can be referred to improve our knowledge concerning the mechanism involved in $\mathrm{TH}$ regulating liver lipid and carbohydrate homeostasis. Moreover, this might be helpful in advising the development of $\mathrm{TH}$ analogues to treat NAFLD and related diseases such as metabolism syndrome.

\section{Methods}

\section{Animals}

Animals were maintained and experiments were performed according to protocols approved by the Animal Care and Use Committees of Institute for Nutritional Sciences. 10 to12-week old male C57BL/6 mice were used in all cases. Mice were rendered hypothyroid by the addition of $0.1 \%$ methimazole (MMI) and $1 \% \mathrm{NaClO}_{4}$ (Sigma, St. Louis, $\mathrm{MO}$ ) in their drinking water for 28 days [37]. As indicated, some animals from this group were injected intraperitoneally with $5 \mu \mathrm{g}$ T3/20 g body weight per day for 5 days, on day 23-27 of $\mathrm{MMI} / \mathrm{NaClO}_{4}$ treatment. Mice were euthanized on the 28th day of MMI treatment or $24 \mathrm{~h}$ after their last T3 injection by exsanguination under anesthesia on day 28. Control mice received no treatment [18]. The treatment was also illustrated schematically in Additional file 4: Figure S4. We evaluated the serum T3 and hepatic Dio1 transcript levels confirmed the establishment of intended hypothyroid status in MMI-treated mice (Additional file 1: Figure S1A and B).

\section{Liver sample collection, fatty acid extraction and FAME preparation}

Mice were euthanized under anesthesia. The dissected liver was cut into several small pieces and immediately frozen in liquid nitrogen or fixed in polyformalin for histological analysis. The liver, weighing 14-18 mg, was first homogenized in ice cold PBS added with internal standard. Fatty acids were extracted by hexane and isopropanol and then incubated with a mixture of methanol and sulfuric acid to produce fatty acid methyl esters (FAMEs). FAMEs were separated by gas chromatography (Agilent 6890 GC; SP-2560 capillary column: $100 \mathrm{~m} \times 0.25 \mathrm{~mm}$ internal diameter $\times 0.2 \mu \mathrm{m}$ film; Supelco). Individual FAMEs were identified by positive chemical ionization with the use of methane as the reagent gas (Agilent 5975B) [38,39].

\section{Real-time RT-PCR and western blot analysis}

Total RNA was isolated by using TRIzol reagent (Invitrogen, USA) according to the manufacturer's instructions. The isolated total RNA was reverse-transcribed by using 
PrimeScript RT reagent Kit (TaKaRa, Shiga, Japan). Realtime PCR was performed on an ABI 7900 Real-Time PCR System (Applied Biosystems). Specific primers for Realtime PCR were listed in Additional file 5: Table S1. The gene expression is quantified by normalizing to $18 \mathrm{~s}$.

Western Blot analysis was performed as described before [40]. Anti-SCD-1 (Santa Cruz Biotechnology, Santa Cruz, CA), anti-FASN (BD, Biosciences PharMingen, San Diego, CA, USA), anti-ACC1 (Cell Signaling Technology, Danvers, MA), anti- $\beta$-Actin (Sigma), anti- $\alpha$-Tubulin (Sigma), and anti-GYS2 (Cell Signaling Technology) antibodies were used as primary antibodies.

\section{Histological analysis}

To stain the neutral lipid, liver specimens were frozen in liquid nitrogen and cryostat sections were used for Oil Red O staining. For immunohistochemical staining, liver specimens were fixed in $10 \%$ neutral buffered formalin and subsequently embedded in paraffin for section. Periodic acid-Schiff (PAS) staining was used to indicate the liver glycogen.

\section{Triglyceride content, glycogen content and serum T3 level determination}

Hepatic triglyceride content was determined by Wako triglyceride lab assay kit (Wako Pure Chemical Industries, Osaka, Japan). Hepatic glycogen content was measured by Glycogen assay kit (Keygen Biotech, Nanjing, China). Blood samples were centrifuged to prepare serum and stored at $-80^{\circ} \mathrm{C}$. Serum total T3 concentrations were determined in Ruijin Hospital affiliated to Shanghai Jiaotong University.

\section{Plasmid, transfection and luciferase assay}

For construction of the luciferase reporter plasmid, the 1.2 kbps promoter region of glycogen synthase 2 was amplified from mouse DNA by PCR and inserted into the pGL3-basic vector, which was designated as GYS2luc. Transfection was performed using Lipofectamine 2000 (Invitrogen, Carlsbad, CA, USA). GYS2-luc or Pal-luc was co-transfected with TR $\alpha$ or TR $\beta$ expression plasmid. $12 \mathrm{~h}$ later, T3 was added at indicated dose. Luciferase assays were performed by using the Dual-Luciferase Reporter Assay System (Promega, Madison, WI, USA) as described previously [18]. Luciferase activity was measured on a luminometer (Berthold Technologies, Bad Wildbad, Germany).

\section{Calculations and statistics}

Summary data for the fatty acid classes, their relative quantities to liver weight percent and mole percent (percentage of each fatty acid of the total fatty acids we determined by GC-MS) were calculated. The results were expressed as means \pm SEM. A nonparametric Wilcoxon signed-rank test was used for two-group comparison. When three groups were compared, analysis of variance was used. A $P$ value of 0.05 or less was regarded as statistically significant.

\section{Additional files}

\begin{abstract}
Additional file 1: Figure S1. Serum T3 levels and hepatic type 1 iodothyronine deiodinase (Dio1) mRNA determination. (A) Serum T3 levels were determined in control group (Ctrl), hypothyroid group (Hypo) and T3-treated group (T3). $n=5$ in each group. Error bars represent the SEM. The serum T3 level of Hypo mice was below $0.25 \mathrm{ng} / \mathrm{ml}$, the lower limit of detection. (B) RT-PCR analysis of Diol gene expression in mouse livers from control group (Ctrl), hypothyroid group (Hypo) and T3-treated group (T3). 18s amplified in parallel served as internal reference. $n=6 \sim 8$ in each group. ${ }^{*} \mathrm{P}<0.05$ vs. Ctrl, ${ }^{\ddagger} \mathrm{P}<0.05$ vs. Hypo.
\end{abstract}

Additional file 2: Figure S2. Schematic of different treatments and designation of groups. Mice of indicated age were treated with $0.1 \%$ $\mathrm{MMl}$ and $1 \% \mathrm{NaClO} 4$ in their drinking water for a total of 28 days (hypothyroid), not treated (control), or treated with $0.1 \% \mathrm{MMI}$ and $1 \%$ $\mathrm{NaClO} 4$ in their drinking water for 28 days and injected with $5 \mu \mathrm{g} \mathrm{T3/20} \mathrm{g}$ body weight per day at 24-h intervals on day 23-27 (T3-treated). Twenty-four hours after the last T3 injection, on day 28 , mice were euthanized by exsanguination, and serum and tissues were collected.

Additional file 3: Figure S3. Hepatic ACC1 and FASN mRNA levels. RT-PCR analysis of ACC1 and FASN gene expression in mouse livers from control group (Ctrl), hypothyroid group (Hypo) and T3-treated group (T3). 18 s amplified in parallel served as internal reference. $n=6 \sim 8$ in each group. ${ }^{*} P<0.05$ vs. $C$ trl, ${ }^{\ddagger} \mathrm{P}<0.05$ vs. Hypo.

Additional file 4: Figure S4. Determination of TR effect on the promoter activity of GYS2. 293T were cotransfected with a reporter containing GYS2 promoter (GYS2-luc) or positive control of thyroid hormone respone element (pal-luc), and TRa or TR $\beta$ expression plasmids as indicated. 12 hours later, 100nM T3 was added into the medium as indicated. Luciferase activity was determined 24 hours after T3 was added. Error bars represent the SEM of three independent experiments. ***P $<0.05$ Ctrl vs T3 (100 nM).

Additional file 5: Table S1. Specific primers for Real-time PCR.

\section{Abbreviations}

ACC1: Acetyl-CoA carboxylase 1; Dio1: Type 1 iodothyronine deiodinase; DNL: De novo lipogenesis; FAMEs: Fatty acid methyl esters; FASN: Fatty acid synthase; GC-MS: Gas chromatography-mass spectrometry; GYS2: Glycogen synthase; MMI: Methimazole; MUFA: Mono unsaturated fatty acid; NAFL: Non-alcoholic fatty liver; NASH: Non-alcoholic steatohepatitis; NFALD: Non-alcoholic fatty liver diseases; PAS: Periodic acid-Schiff; PUFA: Poly unsaturated fatty acid; RTH: Resistance to thyroid hormone; RT: Reverse transcription; SCD-1: Stearoyl-CoA desaturase-1;

SFA: Saturated fatty acids; TFA: Trans fatty acid; TH: Thyroid hormone; T4: Thyroxine; T3: Triiodothyronine; TSH: Thyroid-stimulating hormone; TR: TH receptors; UFA: Unsaturated fatty acid.

\section{Competing interests}

The authors declare that they have no competing interests.

\section{Authors' contributions}

$X Y$ carried out the studies, participated in the animal experiment and FAME preparation, and drafted the manuscript. SH participated in the FAME preparation and GC-MS data extraction. DZ and HX participated in the animal experiment. YCW, HY and JJ participated in the design of the study. HY conceived of the study, and participated in its design and coordination and helped to draft the manuscript. All authors read and approved the final manuscript.

\section{Acknowledgements}

We thank the staffs of the Mass Spectrometry Core Facility at our institute for the technical support on GC-MS. We would also like to thank Dr. Xiaoying Li from Ruijin Hospital affiliated to Shanghai Jiaotong University for providing 
technical support. This work was supported by grants from the Ministry of Science and Technology of China (973 Program 2010CB912500), the National Natural Science Foundation (31371189, 31100550), Shanghai Institutes for Biological Sciences, Chinese Academy of Sciences (SIBS2012004), and CAS/ SAFEA International Partnership Program for Creative Research Teams.

\section{Author details}

'Key Laboratory of Food Safety Research, Institute for Nutritional Sciences, Shanghai Institutes for Biological Sciences, Graduate School of the Chinese Academy of Sciences, Chinese Academy of Sciences, Shanghai 200031, China. ${ }^{2}$ Clinical Research Center of Institute for Nutritional Sciences, Shanghai Institutes for Biological Sciences, Chinese Academy of Sciences, Shanghai 200031, China. ${ }^{3}$ Department of Nutrition, Shanghai Xuhui Central Hospital, Shanghai 200031, China. ${ }^{4}$ Department of Endocrinology and Metabolism, Zhongshan Hospital, Fudan University, Shanghai, China. ${ }^{5}$ Key Laboratory of Food Safety Risk Assessment, Ministry of Health, Beijing, China.

Received: 12 May 2014 Accepted: 15 July 2014

Published: 30 July 2014

\section{References}

1. Baxter JD, Webb P: Thyroid hormone mimetics: potential applications in atherosclerosis, obesity and type 2 diabetes. Nat Rev Drug Discov 2009, 8:308-320.

2. Lazar MA: Thyroid hormone action: a binding contract. J Clin Invest 2003, 112:497-499.

3. Helfand M, Redfern CC: Clinical guideline, part 2. Screening for thyroid disease: an update. American College of Physicians. Ann Intern Med 1998, 129:144-158.

4. Surks Ml, Ortiz E, Daniels GH, Sawin CT, Col NF, Cobin RH, Franklyn JA, Hershman JM, Burman KD, Denke MA, Gorman C, Cooper RS, Weissman NJ, Gorman C, Cooper RS, Weissman NJ: Subclinical thyroid disease: scientific review and guidelines for diagnosis and management. JAMA 2004, 291:228-238.

5. Refetoff S, Weiss RE, Usala SJ: The syndromes of resistance to thyroid hormone. Endocr Rev 1993, 14:348-399.

6. Reddy JK, Rao MS: Lipid metabolism and liver inflammation. II. Fatty liver disease and fatty acid oxidation. Am J Physiol Gastrointest Liver Physiol 2006, 290:G852-G858.

7. Hellerstein MK: De novo lipogenesis in humans: metabolic and regulatory aspects. Eur J Clin Nutr 1999, 53(Suppl 1):S53-S65.

8. Chalasani N, Younossi Z, Lavine JE, Diehl AM, Brunt EM, Cusi K, Charlton M, Sanyal AJ: The diagnosis and management of non-alcoholic fatty liver disease: practice guideline by the American Association for the Study of Liver Diseases, American College of Gastroenterology, and the American Gastroenterological Association. Hepatology 2012, 55:2005-2023.

9. Diraison F, Moulin P, Beylot M: Contribution of hepatic de novo lipogenesis and reesterification of plasma non esterified fatty acids to plasma triglyceride synthesis during non-alcoholic fatty liver disease. Diabetes Metab 2003, 29:478-485.

10. Burra P: Liver abnormalities and endocrine diseases. Best Pract Res Clin Gastroenterol 2013, 27:553-563.

11. Liangpunsakul $\mathrm{S}$, Chalasani $\mathrm{N}$ : Is hypothyroidism a risk factor for non-alcoholic steatohepatitis? J Clin Gastroenterol 2003, 37:340-343.

12. Chung GE, Kim D, Kim W, Yim JY, Park MJ, Kim YJ, Yoon JH, Lee HS: Non-alcoholic fatty liver disease across the spectrum of hypothyroidism. J Hepatol 2012, 57:150-156.

13. Araki $O$, Ying $H$, Zhu $X G$, Willingham $M C$, Cheng $S Y$ : Distinct dysregulation of lipid metabolism by unliganded thyroid hormone receptor isoforms. Mol Endocrinol 2009, 23:308-315.

14. Liu YY, Heymann RS, Moatamed F, Schultz JJ, Sobel D, Brent GA: A mutant thyroid hormone receptor alpha antagonizes peroxisome proliferator-activated receptor alpha signaling in vivo and impairs fatty acid oxidation. Endocrinology 2007, 148:1206-1217.

15. Yen PM: Physiological and molecular basis of thyroid hormone action Physiol Rev 2001, 81:1097-1142.

16. Perra A, Simbula G, Simbula M, Pibiri M, Kowalik MA, Sulas P, Cocco MT, Ledda-Columbano GM, Columbano A: Thyroid hormone (T3) and TRbeta agonist GC-1 inhibit/reverse nonalcoholic fatty liver in rats. FASEB J 2008, 22:2981-2989.
17. Cable EE, Finn PD, Stebbins JW, Hou J, Ito BR, van Poelje PD, Linemeyer DL, Erion MD: Reduction of hepatic steatosis in rats and mice after treatment with a liver-targeted thyroid hormone receptor agonist. Hepatology 2009, 49:407-417.

18. Song Y, Shan S, Zhang Y, Liu W, Ding W, Ren W, Xia H, Li X, Zhang Q, Zhao L, Li X, Yan J, Ying H: Ligand-dependent corepressor acts as a novel corepressor of thyroid hormone receptor and represses hepatic lipogenesis in mice. J Hepatol 2012, 56:248-254.

19. Musso G, Gambino R, Cassader M: Recent insights into hepatic lipid metabolism in non-alcoholic fatty liver disease (NAFLD). Prog Lipid Res 2009, 48:1-26.

20. Zavacki AM, Ying H, Christoffolete MA, Aerts G, So E, Harney JW, Cheng SY, Larsen PR, Bianco AC: Type 1 iodothyronine deiodinase is a sensitive marker of peripheral thyroid status in the mouse. Endocrinology 2005, 146:1568-1575.

21. Wu JH, Lemaitre RN, Imamura F, King IB, Song $X$, Spiegelman D, Siscovick DS, Mozaffarian D: Fatty acids in the de novo lipogenesis pathway and risk of coronary heart disease: the Cardiovascular Health Study. Am J Clin Nutr 2011, 94:431-438.

22. Park MY, Mun ST: Dietary carnosic acid suppresses hepatic steatosis formation via regulation of hepatic fatty acid metabolism in high-fat diet-fed mice. Nutr Res Pract 2013, 7:294-301.

23. Li ZZ, Berk M, McIntyre TM, Feldstein AE: Hepatic lipid partitioning and liver damage in nonalcoholic fatty liver disease ROLE OF STEAROYL-CoA DESATURASE. J Biol Chem 2009, 284:5637-5644.

24. Ntambi JM, Miyazaki M: Regulation of stearoyl-CoA desaturases and role in metabolism. Prog Lipid Res 2004, 43:91-104.

25. Feng $X$, Jiang $Y$, Meltzer $P$, Yen PM: Thyroid hormone regulation of hepatic genes in vivo detected by complementary DNA microarray. Mol Endocrinol 2000, 14:947-955.

26. Flores-Morales A, Gullberg H, Fernandez L, Stahlberg N, Lee NH, Vennstrom B, Norstedt G: Patterns of liver gene expression governed by TR beta. Mol Endocrinol 2002, 16:1257-1268.

27. Sinha RA, You SH, Zhou J, Siddique MM, Bay BH, Zhu XG, Privalsky ML, Cheng SY, Stevens RD, Summers SA, Newgard CB, Lazar MA, Yen PM, Newgard CB, Lazar MA, Yen PM: Thyroid hormone stimulates hepatic lipid catabolism via activation of autophagy. J Clin Invest 2012, 122:2428-2438.

28. Jackson-Hayes $L$, Song $S$, Lavrentyev EN, Jansen MS, Hillgartner FB, Tian L, Wood PA, Cook GA, Park EA: A thyroid hormone response unit formed between the promoter and first intron of the carnitine palmitoyltransferase-lalpha gene mediates the liver-specific induction by thyroid hormone. J Biol Chem 2003, 278:7964-7972.

29. Larque E, Zamora S, Gil A: Dietary trans fatty acids in early life: a review. Early Hum Dev 2001, 65(Suppl):S31-S41.

30. Hashimoto K, Ishida E, Miura A, Ozawa A, Shibusawa N, Satoh T, Okada S, Yamada M, Mori M: Human stearoyl-CoA desaturase 1 (SCD-1) gene expression is negatively regulated by thyroid hormone without direct binding of thyroid hormone receptor to the gene promoter. Endocrinology 2013, 154:537-549.

31. Waters KM, Miller CW, Ntambi JM: Localization of a negative thyroid hormone-response region in hepatic stearoyl-CoA desaturase gene 1. Biochem Biophys Res Commun 1997, 233:838-843.

32. Dobrzyn P, Dobrzyn A, Miyazaki M, Cohen P, Asilmaz E, Hardie DG, Friedman JM, Ntambi JM: Stearoyl-CoA desaturase 1 deficiency increases fatty acid oxidation by activating AMP-activated protein kinase in liver. Proc Natl Acad Sci U S A 2004, 101:6409-6414.

33. Erion MD, Cable EE, Ito BR, Jiang H, Fujitaki JM, Finn PD, Zhang BH, Hou J, Boyer SH, van Poelje PD, Linemeyer DL: Targeting thyroid hormone receptor-beta agonists to the liver reduces cholesterol and triglycerides and improves the therapeutic index. Proc Natl Acad Sci U S A 2007 , 104:15490-15495.

34. Puri P, Baillie RA, Wiest MM, Mirshahi F, Choudhury J, Cheung O, Sargeant C, Contos MJ, Sanyal AJ: A lipidomic analysis of nonalcoholic fatty liver disease. Hepatology 2007, 46:1081-1090.

35. Vujovic M, Nordstrom K, Gauthier K, Flamant F, Visser TJ, Vennstrom B, Mittag J: Interference of a mutant thyroid hormone receptor alpha1 with hepatic glucose metabolism. Endocrinology 2009, 150:2940-2947.

36. Forhead AJ, Cutts S, Matthews PA, Fowden AL: Role of thyroid hormones in the developmental control of tissue glycogen in fetal sheep near term. Exp Physiol 2009, 94:1079-1087. 
37. Marsili A, Ramadan W, Harney JW, Mulcahey M, Castroneves LA, Goemann IM, Wajner SM, Huang SA, Zavacki AM, Maia AL, Dentice M, Salvatore D, Silva JE, Larsen PR: Type 2 iodothyronine deiodinase levels are higher in slow-twitch than fast-twitch mouse skeletal muscle and are increased in hypothyroidism. Endocrinology 2010, 151:5952-5960.

38. Zong G, Zhu J, Sun L, Ye X, Lu L, Jin Q, Zheng H, Yu Z, Zhu Z, Li H, Sun Q, Lin $X$ : Associations of erythrocyte fatty acids in the de novo lipogenesis pathway with risk of metabolic syndrome in a cohort study of middle-aged and older Chinese. Am J Clin Nutr 2013, 98:319-326.

39. Huang Y, Zhu M, Li Z, Sa R, Chu Q, Zhang Q, Zhang H, Tang W, Zhang M, Yin $\mathrm{H}$ : Mass spectrometry-based metabolomic profiling identifies alterations in salivary redox status and fatty acid metabolism in response to inflammation and oxidative stress in periodontal disease. Free Radic Biol Med 2014, 70C:223-232.

40. Zhang D, Li X, Chen C, Li Y, Zhao L, Jing Y, Liu W, Wang X, Zhang Y, Xia H, Chang Y, Gao X, Yan J, Ying H: Attenuation of p38-mediated miR-1/133 expression facilitates myoblast proliferation during the early stage of muscle regeneration. PLoS One 2012, 7:e41478.

doi:10.1186/2045-3701-4-38

Cite this article as: Yao et al: Regulation of fatty acid composition and lipid storage by thyroid hormone in mouse liver. Cell \& Bioscience 2014 4:38.

\section{Submit your next manuscript to BioMed Central and take full advantage of:}

- Convenient online submission

- Thorough peer review

- No space constraints or color figure charges

- Immediate publication on acceptance

- Inclusion in PubMed, CAS, Scopus and Google Scholar

- Research which is freely available for redistribution 